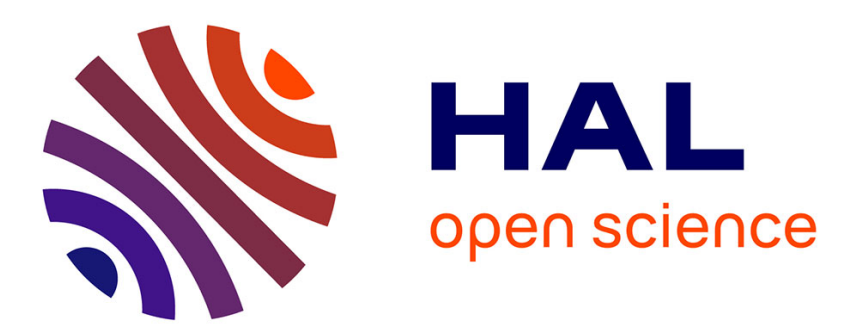

\title{
Surface polarization, rumpling, and domain ordering of strained ultrathin BaTiO 3 (001) films with in-plane and out-of-plane polarization
}

Jelle Dionot, Grégory Geneste, Claire Mathieu, Nick Barrett

\section{- To cite this version:}

Jelle Dionot, Grégory Geneste, Claire Mathieu, Nick Barrett. Surface polarization, rumpling, and domain ordering of strained ultrathin $\mathrm{BaTiO} 3$ (001) films with in-plane and out-of-plane polarization. Physical Review B: Condensed Matter and Materials Physics (1998-2015), 2014, 90, pp.014107. 10.1103/PhysRevB.90.014107 . cea-01376738

\section{HAL Id: cea-01376738 \\ https://hal-cea.archives-ouvertes.fr/cea-01376738}

Submitted on 5 Oct 2016

HAL is a multi-disciplinary open access archive for the deposit and dissemination of scientific research documents, whether they are published or not. The documents may come from teaching and research institutions in France or abroad, or from public or private research centers.
L'archive ouverte pluridisciplinaire HAL, est destinée au dépôt et à la diffusion de documents scientifiques de niveau recherche, publiés ou non, émanant des établissements d'enseignement et de recherche français ou étrangers, des laboratoires publics ou privés. 


\title{
Surface polarization, rumpling, and domain ordering of strained ultrathin $\mathrm{BaTiO}_{3}(001)$ films with in-plane and out-of-plane polarization
}

\author{
Jelle Dionot, ${ }^{1}$ Grégory Geneste, ${ }^{2, *}$ Claire Mathieu, ${ }^{1}$ and Nick Barrett ${ }^{1}$ \\ ${ }^{1}$ CEA, DSM/IRAMIS/SPEC, F-91191 Gif-Sur-Yvette Cedex, France \\ ${ }^{2}$ CEA, DAM, DIF, F-91297 Arpajon, France
}

(Received 23 March 2014; revised manuscript received 25 June 2014; published 23 July 2014)

\begin{abstract}
$\mathrm{BaTiO}_{3}$ ultrathin films (thickness $\approx 1.6 \mathrm{~nm}$ ) with in- and out-of-plane polarization are studied by first-principles calculations. Out-of-plane polarization is simulated using the method proposed by Shimada et al. [Phys. Rev. B 81, 144116 (2010)], which consists in building a supercell containing small domains with alternating up and down polarization. This allows one to investigate the properties of defect free $\mathrm{BaTiO}_{3}$ ultrathin films with polarization perpendicular to the surface, as a function of in-plane lattice constant, i.e., epitaxial strain. The configurations with polarization perpendicular to the surface ( $c$ phase) are found stable under compressive strain, while under tensile strain, the polarization tends to lie in-plane ( $a$ a phase), along [110]. In the $c$ phase, the most stable domain width is predicted to be 1 to 2 lattice constants, and the magnitude of the surface rumpling varies according to the direction of the polarization (upwards versus downwards), though its sign is unchanged, the oxygen anions pointing in all cases outwards. Finally, all the surfaces studied are found to be insulating. Analysis of the atom-projected electronic density of states gives insight into the surface contributions to the electronic structure. An important reduction of the Kohn-Sham band gap is predicted at $\mathrm{TiO}_{2}$ terminations in the $c$ phase $(\approx 1 \mathrm{eV}$ with respect to the $a a$ phase). The Madelung potential at the surface plays the dominant role in modifications of the surface electronic structure.
\end{abstract}

DOI: 10.1103/PhysRevB.90.014107

PACS number(s): 77.80.Dj, 77.80.bn, 77.55.fe

\section{INTRODUCTION}

Simulating the properties of ferroelectric surfaces and thin films from first principles is a complex issue, especially when the polarization $\vec{P}$ is perpendicular to the surface, owing to the depolarizing effects related to the long-range character of the dipole-dipole interaction. It is well known that when $\vec{P}$ is perpendicular to the surface of the film, a surface density of charges $\sigma=\vec{P} \cdot \vec{n}$ appears and gives rise in the bulk of the film to a macroscopic electric field $\vec{E}_{\text {dep }}$, called the depolarizing field. If the polarization in the bulk is constrained to be uniform, this field usually suppresses the ferroelectricity and brings the system back to a nonpolar configuration, the only way to minimize the electrostatic energy of the whole system under such constraint. Preserving ferroelectricity in finite systems requires, therefore, the possibility to screen the depolarizing field, i.e., to create an opposite macroscopic field, that can be of external or internal origin.

The external origin of screening can be related to (i) surface chemistry [1-3] or (ii) the presence of electrodes at the surfaces [4-6]. Metallic electrodes, in particular, allow the accumulation of free charges to compensate the surface density of charges $\sigma$ at the ferroelectric surface [5]. In other words, they create an external electric field $\vec{E}_{0}$ that opposes the depolarizing field $\vec{E}_{\mathrm{dep}}$. In samples without electrodes, however, the screening mechanism more often has an internal origin. Rather than suppressing the polarization, it is often more efficient to form domains with opposite polarization directions, frequent in thin films, or more complex polarization patterns in bulk samples. In thin films exhibiting alternating up and down directions of polarization, the depolarizing field

\footnotetext{
*gregory.geneste@cea.fr
}

inside one domain is mostly compensated by the macroscopic electric field created (mainly) by the two neighboring domains, which have the opposite polarization. The principle of such mechanism is shown in Fig. 1.

This complexity is inherent to the physics of ferroelectric systems and related to the long-range character of the dipoledipole interaction. It makes the simulation of ferroelectric systems (surfaces, slabs, thin films, wires) with polarization perpendicular to the surface quite challenging. Several approaches have been proposed to overcome this difficulty: (i) application of an external electric field [1,7], (ii) freezing ferroelectric displacements in the bulk [8], and more recently (iii) direct simulation of an alternating pattern of up and down polarization [9]. None of these methods is ideal, and all of them have advantages and drawbacks. Methods (i) and (ii) allow simulation of ferroelectric systems with polarization perpendicular to the surface using small supercells, while method (iii) requires a much larger supercell. However, method (i) requires knowledge of the value of the external field to be applied, and in method (ii) the depolarizing field is unscreened in the volume. Method (iii) overcomes these two drawbacks and, applied to $\mathrm{PbTiO}_{3}$ thin films by Shimada et al. [9], is the one that best mimics the real behavior and physical origin of polarization perpendicular to the surface in a thin film. Thus we adopt it in the present work to describe the atomic and electronic structure of freestanding $\mathrm{BaTiO}_{3}(001)$ ultrathin films with polarization perpendicular to their surface, either with $\mathrm{BaO}$ or $\mathrm{TiO}_{2}$ terminations.

The relation between slab thickness $L$ and domain period $2 l$, which minimizes the thermodynamic potential, is normally driven by Kittel's law [10] which states that $L \propto l^{2}$. A change in $L$ therefore requires adjusting $l$ to minimize the free energy and obtain convergence in the ferroelectric properties. This law can be qualitatively understood 


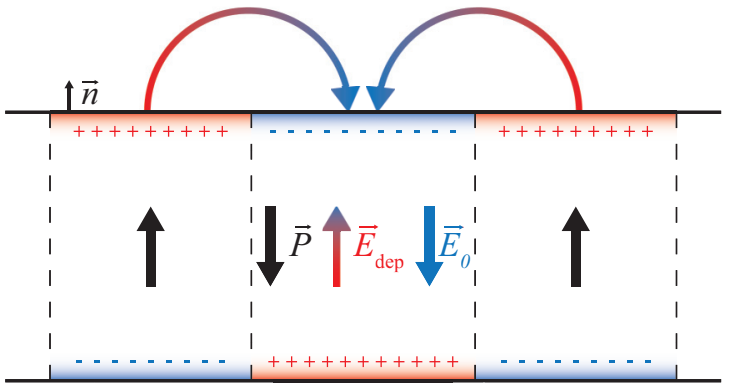

FIG. 1. (Color online) Principle of formation of ferroelectric domains in a thin film with polarization perpendicular to the surface. In the central domain, the depolarizing field $\vec{E}_{\text {dep }}$ (red arrow) is created by the polarization charges accumulated at its surfaces, and mostly compensated by a field $\vec{E}_{0}$ (blue arrow) created by the polarization in the neighboring domains (or, equivalently, by the polarization charges accumulated at the surface of the neighboring domains).

by considering the basic contributions to the energy of a ferroelectric in terms of short/long-range interactions and longitudinal/lateral correlations [11]: Extending the domain width $l$ adds destabilizing (lateral) electrostatic contributions. Thus maintaining the ferroelectric state requires elongating the domain in the direction of the polarization (increase $L$ ), adding stabilizing (longitudinal) electrostatic contributions. When the system size is reduced to the nanoscale, stabilizing a realistic ferroelectric state at very small slab thickness $L$ is difficult because of the increasing impact of the surfaces. Kittel's law therefore probably breaks down in ultrathin films due to surface effects.

In this work, we focus on $\mathrm{BaTiO}_{3}$ (BTO) ultrathin, freestanding films, with thickness $L \approx 1.6 \mathrm{~nm}$. We determine, for this thickness, the optimal domain width $l$ and describe the surface structure of such films. The ferroelectric properties are also studied as a function of lateral lattice constant to simulate the effect of epitaxial strain, and the range of stability of out-of-plane polarized and in-plane polarized thin films is determined. The polarization pattern is analyzed, as well as the surface atomic displacements. Computation of the electronic density of states reveals that all the systems studied, whether they are out-of-plane or in-plane polarized, are insulating.

\section{COMPUTATIONAL DETAILS}

\section{A. Density-functional theory calculations}

We have performed density-functional theory (DFT) calculations [12] using the ABINIT code [13], in the local-density approximation (LDA) (the Perdew-Wang 92 functional is used [14]). We have used Troullier-Martins pseudopotentials [15], which explicitly include as valence electrons the $5 s^{2}, 5 p^{6}$, and $6 s^{2}$ for $\mathrm{Ba}$ (ten electrons), the $3 s^{2}, 3 p^{6}, 4 s^{2}$, and $3 d^{2}$ for $\mathrm{Ti}$ (12 electrons), and the $2 s^{2}$ and $2 p^{4}$ for $\mathrm{O}$ (six electrons). The explicit treatment of semicore electrons is compulsory for a correct description of the ferroelectric instability in barium titanate. A plane-wave cutoff of 40 hartree is used, ensuring convergence of structural and (physical) energetic quantities (better than $1 \mathrm{meV} /$ five-atom cell).
The pseudopotentials have first been validated on bulk BTO: The equilibrium lattice constant of cubic BTO is found at $3.949 \AA$ A. Fixing the lattice constants at the experimental values of the tetragonal phase ( $a=b=3.994 \AA ; c=4.036 \AA)$, the relaxation of the atomic positions in the $P 4 \mathrm{~mm}$ space group provides the following ferroelectric displacements (assuming $\left.\delta z_{\mathrm{Ba}}=0\right): \delta z_{\mathrm{Ti}}=0.055 \AA, \delta z_{\mathrm{O} 1}=-0.118 \AA\left(\mathrm{O}_{1}=\right.$ oxygen belonging to the Ti-O chain parallel to the polarization), and $\delta z_{\mathrm{O} 2}=-0.075 \AA\left(\mathrm{O}_{2}=\right.$ oxygen belonging to the Ti-O chains perpendicular to the polarization). The fully relaxed ferroelectric tetragonal structure has lattice constants $a=b=$ $3.943 \AA$ and $c=3.980 \AA$ with a spontaneous polarization of $24 \mu \mathrm{C} / \mathrm{cm}^{2}$ and ferroelectric displacements $\delta z_{\mathrm{Ti}}=0.043 \AA$, $\delta z_{\mathrm{O}_{1}}=-0.073 \AA$, and $\delta z_{\mathrm{O}_{2}}=-0.051 \AA$. These preliminary results are all in very good agreement with previous LDA calculations $[8,16]$.

Structural optimizations are performed using the BroydenFletcher-Goldfarb-Shanno minimization scheme of ABINIT. The structural optimization is stopped when all the Cartesian components of atomic forces are below $2.0 \times 10^{-4}$ atomic units $(\approx 0.01 \mathrm{eV} / \AA)$.

We have also performed calculations with the SIESTA code $[17,18]$, in the LDA and using Troullier-Martins pseudopotentials (the same electrons are treated as valence as with ABINIT), in order to extract atomic projections of the electronic density of states. The calculations use a numerical atomic orbital basis set that contains from simple $\zeta$ for semicore orbitals (Ba $5 s$, $\mathrm{Ti} 3 s$ ) up to triple $\zeta$ for the more external valence shells (Ti $4 s$, Ti $3 d$, O $2 p$ ). Polarization orbitals are also included. The energy cutoff defining the range of orbitals is $0.001 \mathrm{Ry}$, while that defining the real-space grid is $400 \mathrm{Ry}$.

\section{B. Methodology}

We consider nine-layer BTO slabs with (001) surfaces. This corresponds to freestanding ultrathin films of thickness $\approx 1.6 \mathrm{~nm}$.

For the simulation of films with polarization perpendicular to the surface, we use the method proposed by Shimada et al. [9]: a supercell containing a nine-layer BTO slab is constructed, with the surfaces perpendicular to the $z$ axis. Along an in-plane direction, namely, $y$, the supercell contains $N$ ( $N$ is even) BTO unit cells, and only one unit cell along the $x$ direction. Periodic boundary conditions are applied in the three directions, and a vacuum layer separates the slab from its periodic images. We simulate $\mathrm{BaO}$ and $\mathrm{TiO}_{2}$ terminations. The values of $N$ are 2, 4, and 6 .

The simulated supercells contain $46(N=2), 92(N=4)$, and $138(N=6)$ atoms in the case of the $\mathrm{TiO}_{2}$ termination, and $44(N=2), 88(N=4)$, and $132(N=6)$ atoms in the case of the $\mathrm{BaO}$ termination. The dimensions of the supercells are $L_{x} \times L_{y} \times L_{z}$, with $L_{x}=a, L_{y}=N a$, and $L_{z}=30 \AA$. $a$ is the lateral lattice constant. $L_{z}=30 \AA$ provides a vacuum layer of $\approx 14 \AA$ between the periodic images of the slabs.

The $k$-point sampling of the first Brillouin zone (first BZ) used for the structural optimization is $6 \times 3 \times 1(N=2)$, $6 \times 2 \times 1(N=4)$, and $6 \times 1 \times 1(N=6)$. Once optimized, the final structure is recomputed using denser samplings that exactly correspond to the same $k$-point density in the first BZ, namely, $12 \times 6 \times 1(N=2), 12 \times 3 \times 1(N=4)$, and 


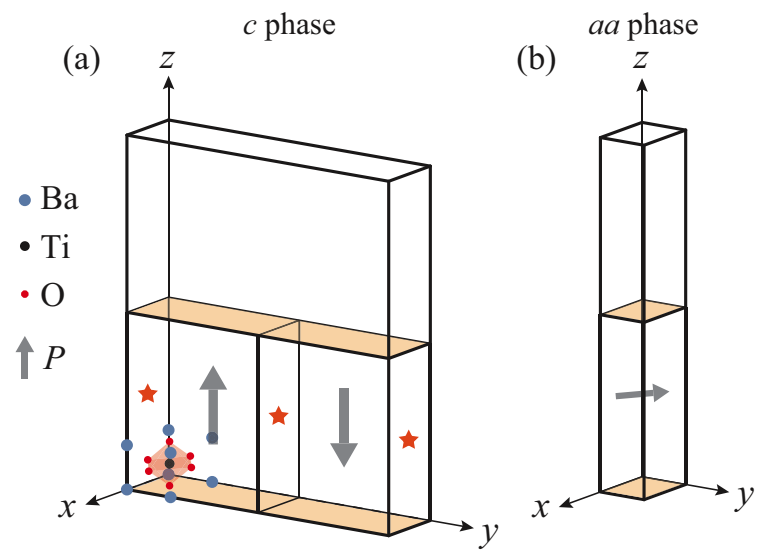

FIG. 2. (Color online) (a) Initial configuration used for the simulation of the $c$ phase. The supercell is $N$ lattice constants long along $y$ and 1 along $x$. The whole supercell is not polar. (b) Initial configuration used for the simulation of the $a a$ phase. The supercell is one lattice constant long along $x$ and $y$ and the supercell is polar. The gray arrows symbolize the initial displacements imposed to the $\mathrm{Ti}$ atoms (it has the same direction as the polarization initially imposed). The orange star shows the location of inversion centers.

$12 \times 2 \times 1(N=6)$. The total energies are extracted from these final calculations.

Structural relaxation of such configurations is conducted by starting from initial displacements along $+z$ on the $\mathrm{Ti}$ atoms with $y_{\mathrm{Ti}}<L_{y} / 2$, and along $-z$ on the $\mathrm{Ti}$ atoms with $y_{\mathrm{Ti}}>L_{y} / 2$, i.e., one upwards and one downwards pointing polarization, allowing the first half of the supercell $(0<y<$ $L_{y} / 2$ ) to develop a polarization along $+z$, and the second half $\left(L_{y} / 2<y<L_{y}\right)$ a polarization along $-z$. After structural optimization, the system might thus form domains of width $N / 2(=1,2$ or 3$)$ unit cells, with alternating up and down polarizations, separated from each other by domain walls. We show below that the existence of such configurations depends on the lateral lattice constant $a$. The configurations exhibiting such domain alternance, with polarization globally perpendicular to the surface in each domain will be denoted as the " $c$ phase." A view of the initial configuration for the simulation of the $c$ phase is shown in Fig. 2(a).

It is important to emphasize that, even though the configurations of the $c$ phase exhibit two ferroelectric domains, with polarizations along $+z$ and $-z$, the whole supercell is not polar, because the dipolar moments of the two domains exactly compensate each other. This is made possible by the symmetry constraints (the initial configuration contains inversion centers located at the center of the domain walls). The whole supercell carries in fact a quadrupole and not a dipole. Since the electric field generated by a quadrupolar distribution of charges decreases with distance much more rapidly than a dipolar one, there is no spurious long-range macroscopic external electric field in the vacuum. We have checked this crucial point by recomputing one of the configurations ( $a=3.80 \AA$, $N=6, \mathrm{TiO}_{2}$ termination) with an increased dimension along $z: L_{z}=45 \AA$, corresponding to a vacuum layer of $\approx 30 \AA$. Increasing the vacuum thickness by $15 \AA$ changes the total energy of the supercell by less than $0.01 \mathrm{eV}$. Moreover, the macroscopic potential along $z$ for one of these configurations is represented in Fig. 11 and exhibits in the vacuum a flat profile when averaged over planes parallel to the surface at fixed $z$. This highlights the absence of a long-range external electric field in the vacuum (see the right-hand side inset of Fig. 11).

We also compute films with in-plane polarization. In that case, a $1 \times 1$ surface unit cell is sufficient (note that this constrains the system to adopt a uniform polarization, thus $90^{\circ}$ domain walls and an in-plane zigzag polarization pattern cannot be observed). The $k$-point sampling is accordingly increased to $6 \times 6 \times 1$ for the structural relaxation. Here again, the final structure is recomputed using a $12 \times 12 \times 1$ mesh to get a total energy that corresponds to a $k$-point density identical to that eventually used for the $c$ phase. We focus on the most stable direction for the in-plane polarization, i.e., [110] [3]. These configurations will be denoted as the "aa phase." A schematic view is shown in Fig. 2(b).

Finally, nonpolar (paraelectric) slabs are also computed as reference systems, using the same $1 \times 1$ surface unit cell and the same $6 \times 6 \times 1$ Brillouin zone sampling for structural relaxation (increased to $12 \times 12 \times 1$ to recompute the final structure). They are denoted in the following as the " $p$ phase."

The in-plane lattice constant $a$ is varied from 3.80 to $4.00 \AA$ in steps of $0.025 \AA$ to simulate the effect of compressive to tensile epitaxial strain. The $c$ phase is computed for $a$ varying from 3.80 to $3.90 \AA$, while the $a a$ phase is computed for $a$ varying from 3.85 to $4.00 \AA$. The reference $p$ phase is computed for all the values of $a$. Calculated with respect to the LDA lattice constant of perfect cubic BTO ( $a=3.949 \AA)$, the lattice constants $3.80,3.90$, and $4.00 \AA$ correspond, respectively, to epitaxial strains of $\approx-3.8 \%, \approx-1 \%$, and $\approx+1.3 \%$.

We denote $P^{+}$as polarization oriented out from the surface, and $P^{-}$as polarization oriented into the surface.

A set of bulk calculations have also been performed, on a five-atom unit cell, to simulate the effect of an epitaxial strain on the bulk phases. These calculations follow the method employed, for instance, in Ref. [19]: The lattice constants are fixed in-plane $(a=b)$ and maintained orthogonal, while the third lattice vector $\vec{c}$ is relaxed until the stress tensor components $\sigma_{3}, \sigma_{4}$, and $\sigma_{5}$ vanish (the Voigt notations are used here). This is equivalent to fixing the strain tensor components $\eta_{1}=\eta_{2}=\eta$ (imposed by the substrate), and $\eta_{6}=0$. The atomic positions are also relaxed. In the present case, such calculations have been performed on the bulk phases that are similar to $c$, $a a$, and $p$, namely, $P 4 m m$ (polarization along [001]), Amm2 (polarization along [110]), and $P 4 / \mathrm{mmm}$ (zero polarization). By symmetry, $\vec{c}$ is orthogonal to $\vec{a}$ and $\vec{b}$ in the three cases. Note that in the case of $A m m 2$, the final component $\sigma_{6}$ of the stress tensor is nonzero because the polarization lies along [110]. This is also the case in the slab calculations of the $a a$ phase.

The present calculations allow simulation of films with in-plane (orthorhombic $a$ a phase) and out-of-plane (tetragonal $c$ phase) polarization. In the $a a$ phase the polarization is along [110], while the domains in the $c$ phase exhibit alternating polarizations oriented along [001] separated by $180^{\circ}$ domain walls. These configurations correspond to the phases appearing at high compressive and high tensile strains on the isotherm $T=0 \mathrm{~K}$ of the temperature/misfit strain phase diagrams (the 
so-called Pertsev diagrams [20,21]) of ferroelectric thin films, except that the present state of the $c$ phase in the ultrathin films include the contributions of surfaces and domain walls. These diagrams usually show an intermediate monoclinic phase $(r$ phase), in which the polarization has an in-plane and an outof-plane component, and smoothly rotates from [001] to [110]. The methodology presented here does not allow simulation of such a phase. Thus, we exclude it from the present discussion.

\section{RESULTS}

\section{A. Stability of the $c$ and $a a$ phases as a function of strain}

We compare the stability of the $c$ and $a a$ phases with respect to the reference $p$ phase, by plotting $E_{\text {tot }}(c)-E_{\text {tot }}(p)$ and $E_{\text {tot }}(a a)-E_{\text {tot }}(p)$ as a function of strain (Fig. 3). To compute this difference in the case of the $c$ phase, the total energy of the supercell with $c$ phase is divided by $N$ to allow a direct comparison with the $p$ and $a a$ phases.

Note that the configurations with $c$ phase contain domain walls, which is not the case with $a a$ and $p$ phases. Their energies therefore include contributions from these domain walls. However, their presence is intrinsic to the existence of $c$ phase in these ultrathin films and has to be accounted for to get a relevant description of the properties of ultrathin BTO films.

In general, the $c$ phase is stabilized at low lattice constants $(<3.90 \AA)$ while the $a a$ phase is stabilized at higher lattice constants, in good qualitative agreement with the misfit-strain diagrams of BTO thin films [19,22] and other ferroelectric systems [23], for which the polarization in films tends to lie out-of-plane (in-plane) in the compressive (tensile) part of the phase diagram.

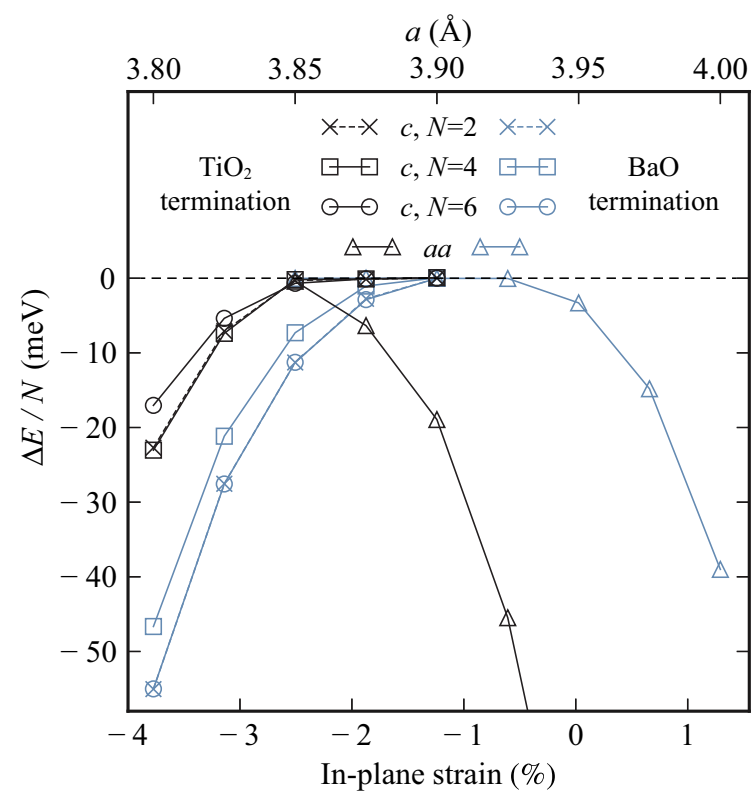

FIG. 3. (Color online) Energy (meV) of the $c$ and $a a$ phases relative to that of the $p$ phase, as a function of epitaxial strain. The transition between $c$ and $a a$ occurs at $\approx-2 \%$ in the case of $\mathrm{TiO}_{2}$-terminated slabs, and $\approx-1 \%$ in the case of $\mathrm{BaO}$-terminated slabs.

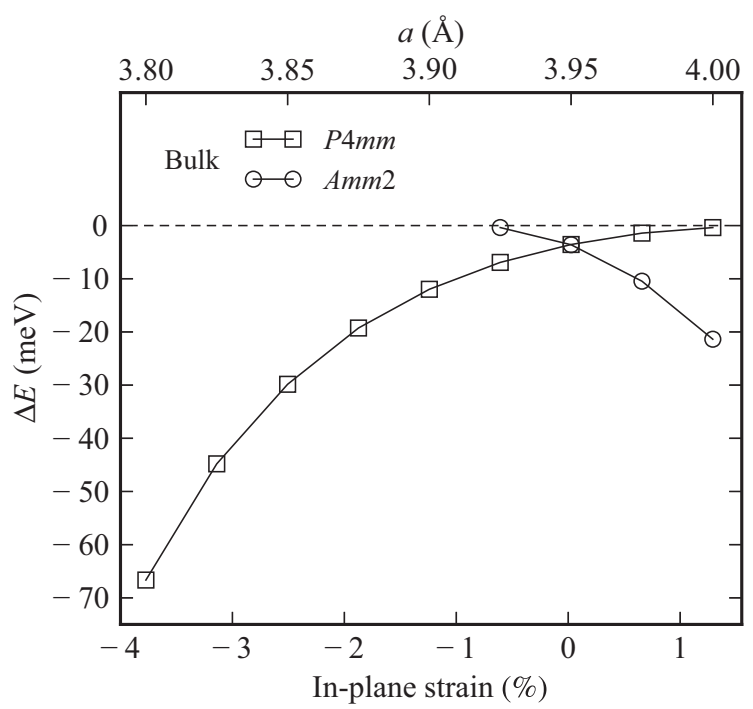

FIG. 4. Energy (meV/unit cell) of the $P 4 \mathrm{~mm}$ (polarization along [001]) and Amm 2 (polarization along [110]) bulk phases, relative to that of the $P 4 / \mathrm{mmm}$ phase, as a function of epitaxial strain. The transition between $P 4 \mathrm{~mm}$ and $A m m 2$ occurs exactly at $0 \%$.

The strain at the transition between the $c$ and $a a$ phases depends on the termination: $\approx-2.5 \%$ (transition $c \rightarrow a a$ ) in the case of $\mathrm{TiO}_{2}$-terminated slabs, and $\approx-1 \%$ (transition $c \rightarrow p$ ) and $\approx-0.5 \%$ (transition $p \rightarrow a a$ ) in the case of $\mathrm{BaO}$-terminated slabs. In the bulk, the transition between the corresponding phases, namely, $P 4 m m$ and $A m m 2$, takes place exactly at $0 \%$ (Fig. 4), illustrating a strong surface effect in thin films on the value of the lattice parameter of the phase transition.

In the $\mathrm{TiO}_{2}$ case, the aa phase is much more stable than the bulk $A m m 2$ phase, reflecting the known tendency of $\mathrm{TiO}_{2}$ surfaces to strongly enhance and stabilize in-plane ferroelectric distortions [3,24]. For instance, at $a=3.95 \AA$, the energy of the $a a$ phase is $\approx-90 \mathrm{meV}$ (for $\approx 4.5$ unit cells, thus $\approx-20 \mathrm{meV} /$ unit cell) whereas that of $A m m 2$ is -3.6 $\mathrm{meV} /$ unit cell. On the other hand, the $c$ phase is less stable than the bulk $P 4 m m$ phase. For instance, at $a=3.85 \AA$, the energy of the $c$ phase is close to zero, whereas that of $P 4 \mathrm{~mm}$ is $-30 \mathrm{meV} / \mathrm{unit}$ cell. The stabilizing of $a a$ and the destabilizing of $c$ results in the transition lattice constant shifted to higher compressive strain, namely, $\approx-2.5 \%$.

In the $\mathrm{BaO}$ case, the $a \mathrm{a}$ phase is destabilized with respect to the bulk $A m m 2$ phase, reflecting, here again, the known tendency of $\mathrm{BaO}$ surfaces to reduce and destabilize in-plane ferroelectric distortions $[3,24]$. The $c$ phase is also destabilized, but less than in the $\mathrm{TiO}_{2}$ case. Since both phases are destabilized, there is no crossover, and the $\mathrm{BaO}$-terminated slabs are no longer ferroelectric over a strain range from $\approx-1 \%$ to $-0.5 \%$.

Moreover, we observe that the equilibrium in-plane lattice constant of the slabs (corresponding to unstressed films) is lower than $3.949 \AA$ in the case of the $\mathrm{TiO}_{2}$ surface: The energy minimum corresponds for this termination to $a \approx 3.91 \AA$ ( $c$ and $p$ phases) [3.92 $\AA$ (phase $a a)$ ], whereas it corresponds to $a \approx 3.94 \AA$ ( $a a$ and $p$ phases) [3.95 $\AA$ ( $c$ phase)] for the $\mathrm{BaO}$ surface. The shift from an equilibrium in-plane lattice 
parameter of $3.95 \AA$ in bulk to lower values in freestanding slabs is obviously a surface effect. Interatomic bond lengths usually tend to be shorter at surfaces due to lower coordination numbers, creating at the surface an extra-usually tensilestress tensor, the so-called surface stress [25]. This effect is weak in the $\mathrm{BaO}$-terminated slabs, but significant in the $\mathrm{TiO}_{2}$-terminated ones, for which it is large enough to shift the equilibrium lattice constant by $\approx 0.03-0.04 \AA$ towards compressive strain values.

In the compressive part ( $c$ phase), the energy of thin films depends on the period of the domain structure. In the case of the $\mathrm{TiO}_{2}$-terminated slab, the most stable structures correspond to $N=2$ and $N=4$, which have very similar energies, while $N=6$ exhibits a higher energy, though still more stable than the nonpolar $p$ phase. In the case of the $\mathrm{BaO}$-terminated slab, the most stable structure corresponds to $N=2$ (domains of one lattice constant width). $N=4$ has a higher energy, though more stable than the nonpolar $p$ phase, and $N=6$, after structural optimization, returns to the same structure as $N=2$ (see Sec. III B). Freestanding BTO thin films of $1.6 \mathrm{~nm}$ thickness therefore develop, under sufficient compressive epitaxial strain, domain patterns with periodicity of two to four lattice constants, i.e., domains of one to two lattice constants width, and polarization perpendicular to their surface.

\section{B. Polarization pattern, atomic surface displacements, and electronic structure of $c$ phase}

\section{Polarization pattern: Closure domain}

In order to inspect the polarization pattern in the ferroelectric domains of the $c$ phase, we first need to define a local polarization in each unit cell. Following Shimada et al. [9], the polarization in unit cell $i$ is defined as

$$
P_{\alpha}(i)=\frac{1}{v_{i}} \sum_{k \in(i), \beta} w_{k} Z_{\alpha \beta}^{*(k)} \delta u_{\beta}(k),
$$

in which the sum is over all the atoms belonging to the cell $(i), v_{i}$ is the volume of cell $(i), w_{k}$ is a weight (between 0 and 1) affected to the atoms that are shared between several unit cells, and $Z_{\alpha \beta}^{*(k)}$ is the Born effective charge tensor of atom $k$, taken to be that of perfect bulk cubic BTO [26]. The atomic displacements $\delta \vec{u}(k)$ are calculated with respect to the positions of the nonpolar $p$ phase (with atomic positions relaxed at the relevant lateral lattice constant). By convention, in the $\mathrm{TiO}_{2}$-terminated slabs, a unit cell is centered on an oxygen atom of a $\mathrm{BaO}$ plane, while in the $\mathrm{BaO}$-terminated slabs, a unit cell is centered on a titanium atom of $\mathrm{a} \mathrm{TiO}_{2}$ plane.

The ferroelectric displacements become larger as the compressive strain is increased, thus in the following we focus on the patterns obtained in the case of the strongest compressive strain $(a=3.80 \AA)$. Figure 5 shows a lateral view in the $(y, z)$ plane of the polarization patterns for the $\mathrm{TiO}_{2}$ and the $\mathrm{BaO}$ terminations. The upper panels with the atom-centered colored arrows show the displacements, and the lower panels give the total, $y$ and $z$ components of the local polarization for each unit cell.

For $N=4$ and $N=6$ in the case of the $\mathrm{TiO}_{2}$-terminated slabs, and for $N=4$ in the case of the $\mathrm{BaO}$-terminated slabs [Figs. 5(b), 5(c), and 5(e)], at the surface near the domain wall, the polarization is not perpendicular to the surface: It turns to form closure domains, as already observed in $\mathrm{PbTiO}_{3}$ [9] and in BTO films between metallic electrodes [6]. For $N=2$ in the case of the $\mathrm{TiO}_{2}$-terminated slabs and for $N=2$ and $N=6$ in the case of the BaO-terminated slabs [Figs. 5(a), 5(d), and 5(f)], the magnitude of the polarization decreases towards the surface, suggesting an incipient closure domain behavior (visible from the lateral atomic displacements in the surface plane), although it is obviously impossible to observe closure for domains one unit cell wide. The formation of closure domains is natural since it allows the system to minimize its electrostatic energy by minimizing the density of polarization charges $\rho_{\text {pol }}$, which are created by the abrupt variations of $\vec{P}$ $\left(\rho_{\mathrm{pol}}=-\vec{\nabla} \cdot \vec{P}\right)$.

The $N=2$ configurations could be considered, at first glance, as antiferroelectric (consecutive to the freezing of the instability at the $X$ point of the Brillouin zone of the parent cubic structure). However, in the present case, stabilization of such patterns obviously results from the screening of the depolarizing field by the neighboring domains, rather than from a bulk antiferroelectricity: Since these configurations also exhibit an incipient closure domain behavior, which is much more developed for larger values of $N$, we prefer considering them as the limit, for small film thicknesses or small domain width, of the alternating up and down ferroelectric configurations obtained at larger $N$. Moreover, antiferroelectric instabilities (such as in $\mathrm{PbZrO}_{3}$ ) are rather complex phenomena $[27,28]$ that differ in nature from what is observed in $\mathrm{BaTiO}_{3}$ films in the present study.

It is interesting to compare the local polarizations to the bulk polarization, obtained using the same lateral lattice constant $a=b$ with full relaxation of the $c$ lattice parameter and internal positions in the tetragonal $P 4 \mathrm{~mm}$ phase. For $a=b=3.80 \AA$, the bulk polarization is $56.5 \mu \mathrm{C} / \mathrm{cm}^{2}$. We see that the local polarization in the films remains much smaller than the corresponding bulk value, despite the strong compressive strain obtained using that lattice constant. In the case of the $\mathrm{TiO}_{2}$ termination, the local polarization reaches $\approx 30-35 \mu \mathrm{C} / \mathrm{cm}^{2}(\approx 60 \%$ of the bulk value) in the middle of the slab, while in the case of the $\mathrm{BaO}$ termination, it is $\approx 41-$ $43 \mu \mathrm{C} / \mathrm{cm}^{2}$ ( $\approx 75 \%$ of the bulk value). At the surfaces, the amplitude of the rotating polarization is even smaller. Smaller compressive strains (i.e., lateral lattice constants between 3.80 and $3.90 \AA$ ) of course yield smaller local polarizations $\vec{P}$, which are almost zero for a lateral lattice constant $3.90 \AA$.

The epitaxial strain corresponding to $a=3.80 \AA$ is $-3.8 \%$. This large value is far from the epitaxial strains usually employed in thin films, although large compressive strains can also be attained using, for instance, $\mathrm{LaAlO}_{3}$ as a substrate. However, we emphasize that it is necessary to obtain appreciable values of the polarization in $c$-phase configurations. At $a=3.90 \AA(-1 \%)$, for instance, the surface effect is so strong that the polarization is almost zero in the $c$ domains. Very large compressive strains are thus a necessary condition to the existence of $c$ phase in ultrathin BTO films.

\section{Atomic out-of-plane displacements at surfaces}

We define the surface rumpling in a given surface unit cell as the quantity $r=\frac{\left\langle z_{\text {cat }}\right\rangle-\left\langle z_{0}\right\rangle}{a},\left\langle z_{\text {cat }}\right\rangle$ being the average $z$ 


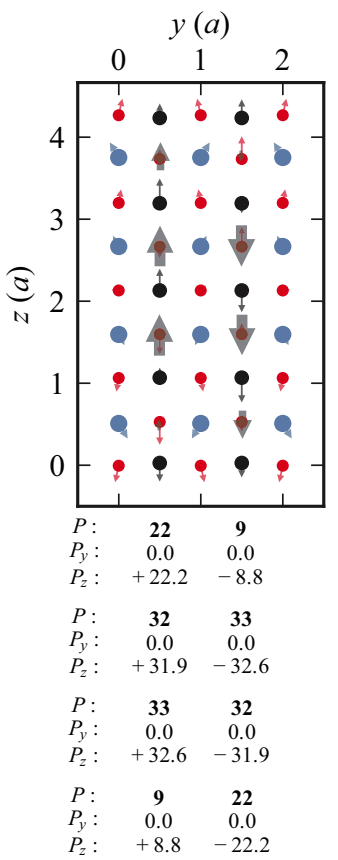

(a) $N=2$

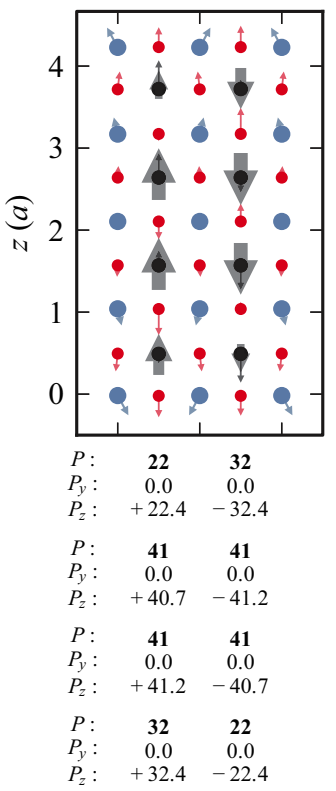

(d) $N=2$

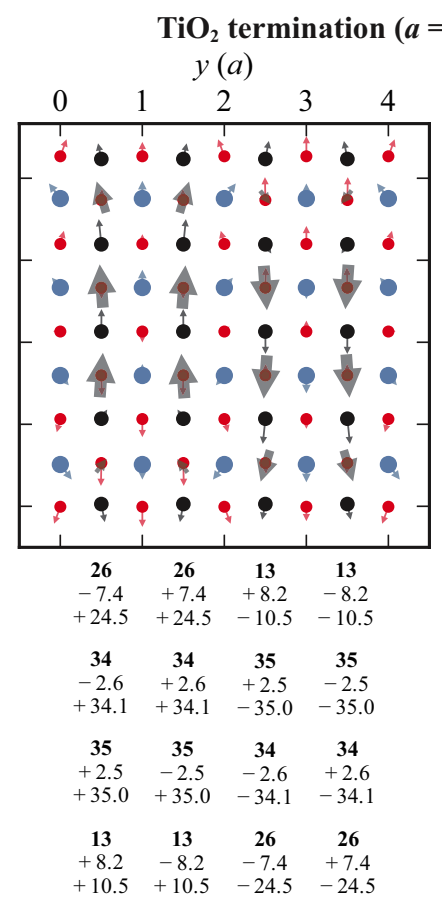

(b) $N=4$

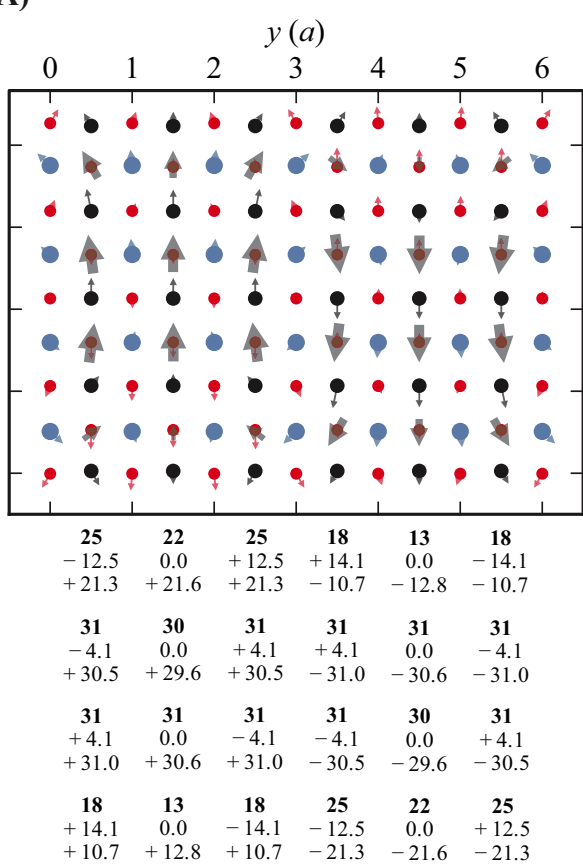

(c) $N=6$

$\mathrm{BaO}$ termination $(a=3.80 \AA)$

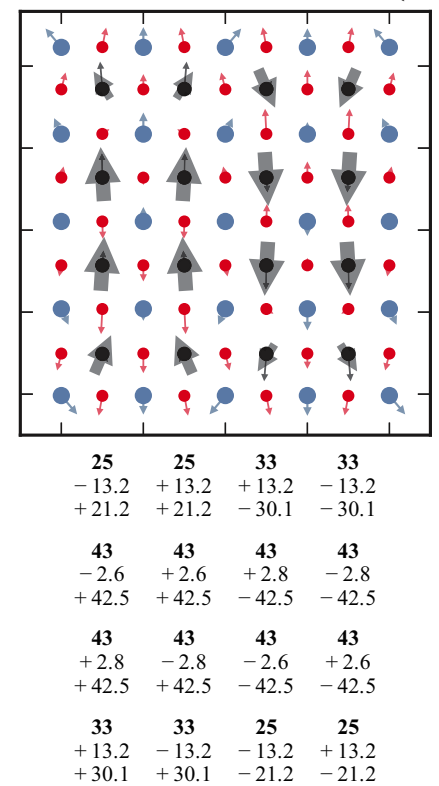

(e) $N=4$

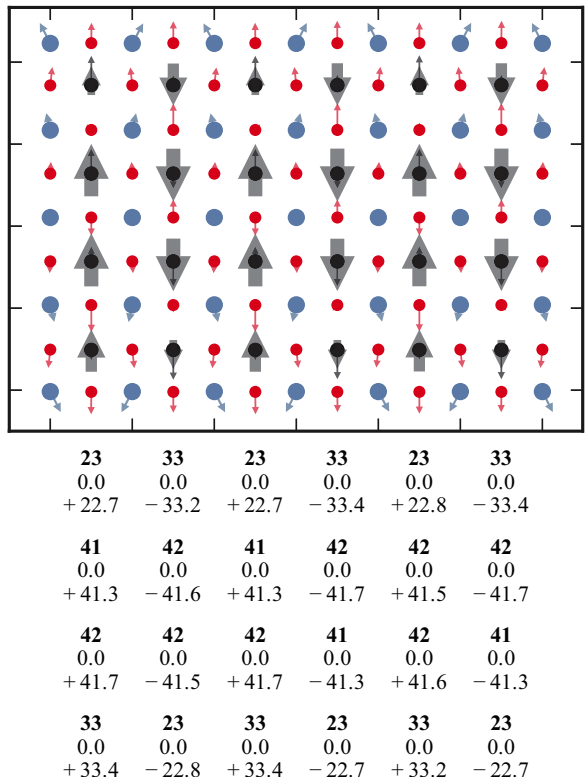

(f) $N=6$

FIG. 5. (Color online) Local polarization patterns of $\mathrm{TiO}_{2}$-terminated [(a)-(c)] and $\mathrm{BaO}$-terminated [(d)-(f)] freestanding thin films in the $c$ phase with lateral lattice constant $3.80 \AA$ and domain periodicity $N$. On the top, the colored circles and arrows show the atoms in the reference $p$ phase and six times the corresponding displacements in the $c$ phase, respectively; the large dark arrows depict the ensuing local polarization vectors. Below each panel, the magnitude $P$, as well as the $y\left(P_{y}\right)$ and $z\left(P_{z}\right)$ components of the local polarization expressed in $\mu \mathrm{C} / \mathrm{cm}^{2}$ are given for each unit cell.

coordinate of the surface cation (either $\mathrm{Ba}$ or $\mathrm{Ti}$ ), and $\left\langle z_{\mathrm{O}}\right\rangle$ that of the oxygen atoms of the surface plane. We express $r$ in percent of the lateral lattice constant $a$. In the case of the $\mathrm{TiO}_{2}$ termination, $\left\langle z_{\text {cat }}\right\rangle=z_{\mathrm{Ti}}$ and $\left\langle z_{\mathrm{O}}\right\rangle$ is the average of the $z$ coordinate of the four $\mathrm{O}$ surrounding Ti. In the case of the $\mathrm{BaO}$ termination, $\left\langle z_{\mathrm{O}}\right\rangle$ is the $z$ coordinate of the surface
$\mathrm{O}$ atom of the unit cell considered, and $\left\langle z_{\text {cat }}\right\rangle$ is the average of the $z$ coordinate of the four Ba surrounding O. Figure 6 shows the rumpling at the $\mathrm{TiO}_{2}$ and $\mathrm{BaO}$ terminations in the case of $N=6$.

The surface rumpling at surfaces of ionic or ionocovalent crystals usually consists of the anions displaced outwards and 


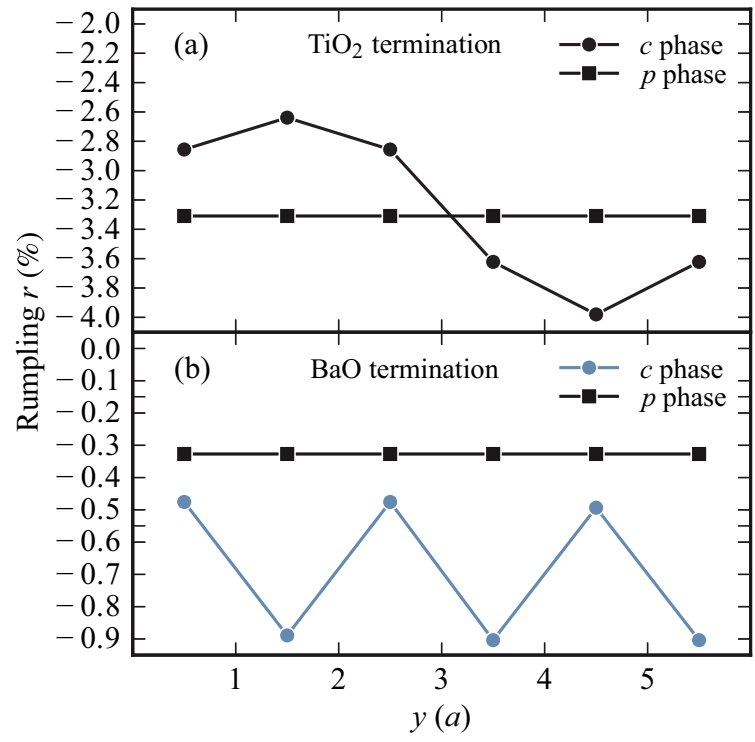

FIG. 6. (Color online) Surface rumpling $r$ for the $c$ and $p$ phases for the $\mathrm{TiO}_{2}$ (a) and $\mathrm{BaO}$ (b) -terminated slab with $a=3.80 \AA$ and $N=6$.

the cations displaced inwards. In the present case, whatever the direction or amplitude of the local polarization, $r$ is always negative. Thus the oxygen atoms move outwards while cations (either $\mathrm{Ba}$ or $\mathrm{Ti}$ ) move inwards. The magnitude of $r$ differs depending on termination $\left(\mathrm{TiO}_{2}\right.$ or $\left.\mathrm{BaO}\right)$ and phase $(c, a a$, or $p$ ).

The rumpling at the surface of the $p$ phase gives an indication about the natural displacements of the surface atoms, i.e., in the absence of ferroelectric induced distortion. Under high compressive strain ( $a=3.80 \AA$ ),$r=-3.31 \%$ for the $\mathrm{TiO}_{2}$ termination, but is much smaller, $-0.33 \%$ for the $\mathrm{BaO}$ termination.

At the surface of the $c$ phase under the same compressive strain $(a=3.80 \AA)$, the ferroelectric displacements modify those of the natural rumpling. For the $\mathrm{TiO}_{2}$ termination, on the $P^{-}$surface the ferroelectric distortion enhances the rumpling which reaches values between -3.6 and $-4.0 \%$, while on the $P^{+}$surface, the rumpling is decreased by the ferroelectricity to between -2.65 and $-2.85 \%$. For the $\mathrm{BaO}$ termination, the rumpling is globally enhanced from $-0.33 \%$ to $\approx-0.5 \%$ at the $P^{+}$surface, and $\approx-0.9 \%$ at the $P^{-}$surface.

\section{Electronic density of states}

In this part, we calculate the electronic density of states (DOS) of the films. In the $c$ phase, the densities of states have been computed in the case of the largest compressive strain $(a=3.80 \AA)$. For the different domain periodicities $(N=2$, 4 , and 6), and for a given termination, they are very similar. Figure 7 shows this DOS in the case of $N=6$ and $a=3.80 \AA$ for the two terminations. By convention, the energy of the highest occupied state $\left(E_{F}\right)$, which corresponds to the valence band maximum in an insulator, is placed at zero. In all the calculations, the system remains an insulator. However, an important difference between the $\mathrm{TiO}_{2}$-terminated and $\mathrm{BaO}$ terminated slabs is seen: The Kohn-Sham band gap is slightly

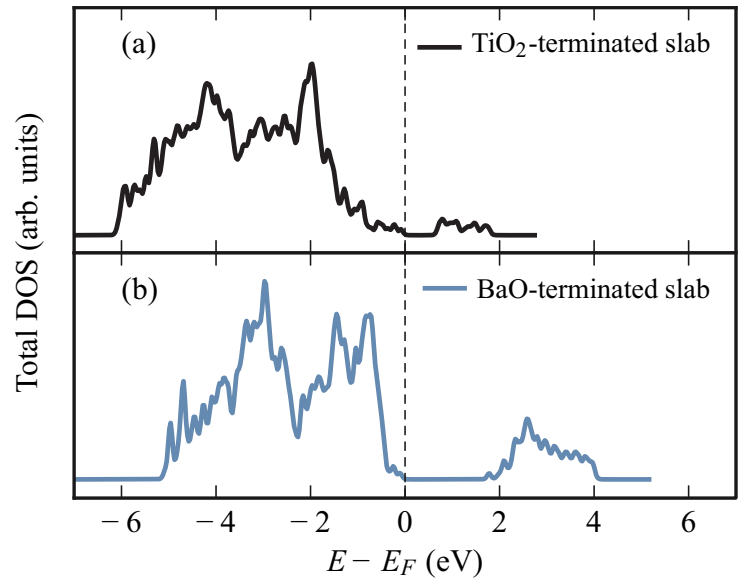

FIG. 7. (Color online) Electronic density of states of the $c$ phase for both terminations, as computed with ABINIT. The Fermi energy $E_{F}$ is at $0 \mathrm{eV}$. $N=6, a=3.80 \AA$. Phases $c$ with $N=2$ and $N=4$ exhibit very similar densities of states.

less than $2 \mathrm{eV}$ in the $\mathrm{BaO}$ case, while it is strongly reduced to $\approx 0.5 \mathrm{eV}$ in the $\mathrm{TiO}_{2}$ case.

In the $\mathrm{TiO}_{2}$ case, the density of states has also been projected on different regions of the supercell (Fig. 8) in order to obtain further insight into the contributions of each part of the system to the electronic structure. These contributions are shown by the thick lines of Fig. 8. The bulk part provides a density of states with a Kohn-Sham band gap larger than that of the slab, as expected (the valence band maximum has a much lower energy), while the two surfaces, $P^{-}$and $P^{+}$, provide densities of state with a very small gap (smaller by $\approx 1.5 \mathrm{eV}$ ). The surfaces are thus responsible for the reduction of the Kohn-Sham band gap of the thin film, due to the presence of electronic surface states above the valence band maximum (VBM) of the bulk. The densities of state at the top of the valence band are very similar for $P^{-}$and $P^{+}$. However, the two surface polarizations differ significantly by their contributions to the bottom of the conduction band [Figs. 8(b) and 8(c)]. Our calculations do not show any evidence of a surface metallization when the polarization is perpendicular to the surface, i.e., in the $c$ phase. This provides indirect confirmation that depolarizing fields are well screened by domain formation in these ultrathin films.

Surface metallization, when observed experimentally, is thus very probably the consequence of the presence of point defects such as oxygen vacancies [29]. Theoretical predictions of metallization of the surface of BTO [8] are more probably due to the use of fixed atomic positions in the bulk creating an unscreened depolarizing field which in turn can induce a surface density of states at the Fermi level.

We denote $\mathrm{O}_{\mathrm{I}}$ and $\mathrm{O}_{\text {II }}$ as the oxygen atoms belonging respectively to the $\mathrm{BaO}$ and $\mathrm{TiO}_{2}$ atomic layers of the slab. Figures 8(b)-8(d) reveal that the surface states at the top of the valence band of the $c$ phase are mainly localized on oxygen atoms of the surface layer, $\mathrm{O}_{\text {II }}$. It also reveals that such surface states exist high in the gap, whatever the direction of $\vec{P}$, i.e., for $P^{+}$and $P^{-}$. 


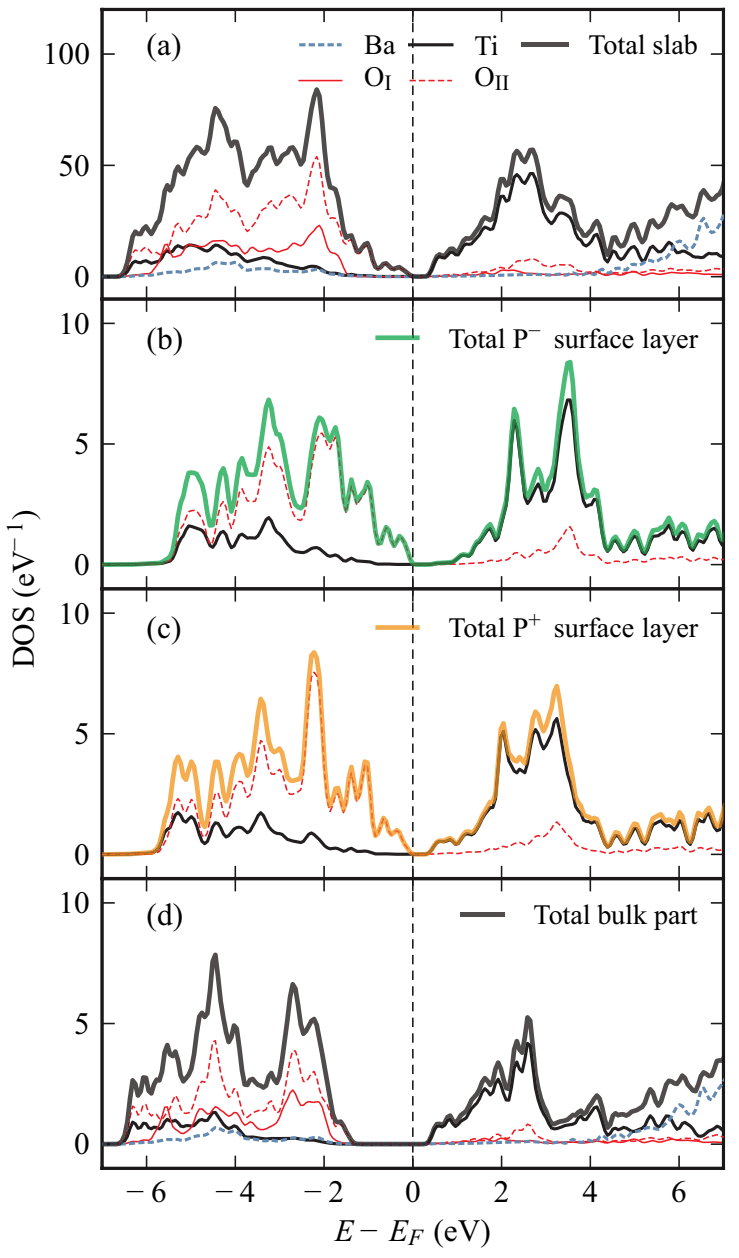

FIG. 8. (Color online) Electronic density of states of the $c$ phase for the $\mathrm{TiO}_{2}$-terminated film, as computed with SIESTA. $N=6, a=$ $3.80 \AA$ A. (a) Total DOS; (b) DOS projected on the first atomic layer at the $P^{-}$surface; (c) DOS projected on the first layer at the $P^{+}$surface; and (d) DOS projected on the bulk part of the slab.

\section{Characteristics of $a$ a phase}

The configurations of the $a a$ phase, which are in-plane polarized, are stabilized with respect to the $c$ phase for strains higher than $\approx-2.5 \%$ in the case of $\mathrm{TiO}_{2}$ surfaces and $\approx-0.5 \%$ in the case of $\mathrm{BaO}$ surfaces. In the $\mathrm{TiO}_{2}$ case, they are more stable than their bulk counterpart (Amm2 phase), while in the $\mathrm{BaO}$ case, they are less stable. The polarization increases with increasing tensile strain, and for a given strain, grows (decreases) from the center of the slab to the $\mathrm{TiO}_{2}(\mathrm{BaO})$ surface. For instance, at $a=4.00 \AA,\|\vec{P}\|$ is $53 \mu \mathrm{C} / \mathrm{cm}^{2}$ at the surface unit cells, and $37 \mu \mathrm{C} / \mathrm{cm}^{2}$ in the central cells in the case of the $\mathrm{TiO}_{2}$-terminated slab. In contrast, it is $27 \mu \mathrm{C} / \mathrm{cm}^{2}$ at the surface unit cells, and $32 \mu \mathrm{C} / \mathrm{cm}^{2}$ in the central cells of the $\mathrm{BaO}$-terminated slab.

The atomic displacements at surfaces correspond to negative rumpling. At the highest tensile strain studied $(a=$ $4.00 \AA$ ), the rumpling is $-2.4 \%$ in the case of the $\mathrm{TiO}_{2}$ terminated slab (weaker than in the $c$ phase) and $-2.0 \%$ in the case of the $\mathrm{BaO}$-terminated slab.

Figure 9 shows electronic density of states of $\mathrm{TiO}_{2}$ - and $\mathrm{BaO}$-terminated slabs in the $a a$ phase, computed at the highest

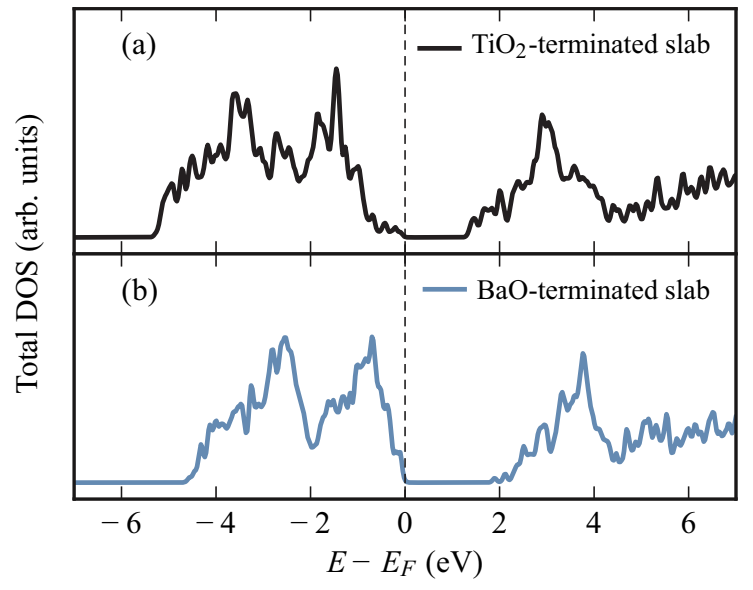

FIG. 9. (Color online) Electronic density of states of the a a phase for both terminations, as computed with ABINIT. The Fermi energy $E_{F}$ is at $0 \mathrm{eV}$. The lateral lattice constant corresponds to the highest tensile strain studied, $a=4.00 \AA$.

tensile strain studied ( $a=4.00 \AA$ ). A Kohn-Sham band gap of $\approx 2 \mathrm{eV}$ is found in the $\mathrm{BaO}$ case, as for the $c$ phase. The Kohn-Sham band gap in the case of the $\mathrm{TiO}_{2}$-terminated slab is reduced to $\approx 1.4 \mathrm{eV}$, which is, however, much higher than the same termination in the $c$ phase. This difference is mainly caused by the surface states at the top of the valence band, which do not extend high into the gap in the tensile case, whereas they exist up to $\approx 1 \mathrm{eV}$ above the bulk VBM at the $\mathrm{TiO}_{2}$ surface of the $c$ phase. The origin of these differences may be in the way that the surface rumpling and ferroelectric distortion interact. The surface rumpling of the $a a$ phase for the $\mathrm{TiO}_{2}$ termination is reduced with respect to the $c$ phase, whereas for the $\mathrm{BaO}$ termination it is enhanced.

\section{DISCUSSION}

\section{A. Screening of the depolarizing field by domain formation}

Our calculations show that freestanding BTO thin films of $1.6 \mathrm{~nm}$ thickness might exhibit ferroelectricity perpendicular to their surface provided a compressive strain is applied to the film. The period of the domain pattern is very short: two lattice constants in the case of the $\mathrm{BaO}$ surface, and two to four lattice constants in the case of the $\mathrm{TiO}_{2}$ surface. $\mathrm{TiO}_{2}$-terminated films with larger period $(N=6)$ are less stable although they are always more stable than the nonpolar phase. For an epitaxial strain of $\approx-1 \%(-2.5 \%)$, the $\mathrm{BaO}$-terminated ( $\mathrm{TiO}_{2}$-terminated) slabs switch to an in-plane polarization, along [110]. The mechanism of screening by formation of stripe domains is thus efficient for freestanding ultrathin BTO films provided compressive strain is applied. The difference in stability of ultrathin ferroelectric films, between $\mathrm{BaO}$ - and $\mathrm{TiO}_{2}$-terminated slabs, is perhaps related to the surface induced electronic states above the VBM. In the case of $\mathrm{TiO}_{2}$, the higher surface DOS can make a stronger contribution to the minimization of the energy than for $\mathrm{BaO}$-terminated slabs.

Junquera and Ghosez have shown that ultrathin BTO films between metallic $\mathrm{SrRuO}_{3}$ electrodes with polarization perpendicular to the surface have a critical thickness, below which ferroelectricity cannot exist in the film under the form of 
a uniform state, due to incomplete screening by the free charges of the metal [5]. Aguado-Puente and Junquera have completed this picture by allowing BTO thin films (also in short-circuit conditions between $\mathrm{SrRuO}_{3}$ metallic electrodes) to form two domains with opposite polarizations, as in the present study [6]. They showed that ferroelectricity perpendicular to the surface might exist in such systems for thicknesses lower than the critical thickness associated with the uniform state, giving insight into the capacity of domain formation to screen the depolarizing electric field in thin films: A combination of domain formation with free charges of metallic electrodes allows such stabilization down to very small thicknesses in BTO, although attention must be paid to possible artifacts of DFT using the LDA approximation, which can give rise to electrons overspilling into the ferroelectric and apparent metallization of the first atomic layers [4].

Here we show that, if the screening power of the metal is removed, ferroelectricity perpendicular to the surface in BTO ultrathin films can be maintained provided strong compressive strain is applied. The ferroelectricity is indeed very weak in the $c$ phase for epitaxial strains close to zero, between the compressive and the tensile part of the phase diagram. Quantum zero-point motions, which in $\mathrm{BaTiO}_{3}$ are related to pronounced anharmonicities [30-32], can also have an impact on the magnitude of the polarization especially when the latter is small. Finally, we note that the magnitude of the local polarization in the $c$ phase is reduced with respect to the bulk in the same epitaxial conditions (between $60 \%$ and $75 \%$ of the bulk value at the center of the domains).

In the study of Shimada et al. performed on $\mathrm{PbTiO}_{3}$ [9], ferroelectricity perpendicular to the surface, under the form of closure domains, is obtained in ultrathin films with a lateral lattice constant set at the LDA theoretical value $(3.867 \AA)$. However, there is a noticeable difference between tetragonal $\mathrm{PbTiO}_{3}$ and $\mathrm{BaTiO}_{3}$ at their theoretical lattice constant: The spontaneous polarization of $\mathrm{PbTiO}_{3}$ is $90 \mu \mathrm{C} / \mathrm{cm}^{2}$ [33] while that of $\mathrm{BaTiO}_{3}$ is $\approx 24 \mu \mathrm{C} / \mathrm{cm}^{2}$, reflecting the known higher spontaneous polarization of $\mathrm{PbTiO}_{3}$. This strong spontaneous polarization allows the appearance of $c$ phase even at the LDA lattice constant.

\section{B. Surface effects in ultrathin ferroelectric films}

Our calculations provide evidence for a strong impact of the surface on the properties of ultrathin ferroelectric films.

First, surface effects modify the stability of the ferroelectric phases with respect to the bulk. In-plane polarized configurations ( $a$ a phase) are stabilized by a $\mathrm{TiO}_{2}$ termination, but destabilized by the $\mathrm{BaO}$ one. Such a tendency has already been observed at BTO(001) surfaces [3] and in nanowires [24]. In contrast, out-of-plane polarization configurations ( $c$ phase) are destabilized by the surface whatever the termination (the $\mathrm{TiO}_{2}$ one more so). As a result of the surface, the lattice constants of transition between the different ferroelectric phases are shifted towards compressive strains.

In the $\mathrm{TiO}_{2}$ case, the transition $c \rightarrow a a$ occurs at $\approx-2.5 \%$. In the $\mathrm{BaO}$ case, the sequence of phases is $c \rightarrow p \rightarrow a a$, at $\approx-1 \%$ and $-0.5 \%$. We point out, however, that the present calculations do not take into account the possibility of a monoclinic $r$ phase at intermediate strain between $c$ and $a a$.
Nevertheless, our calculations show that surface effects may strongly affect the ferroelectric properties, modifying the energy of the phases, the polarization close to the surface and the value of the lattice constant (i.e., epitaxial strain) at which phase transitions are normally observed in bulk or in thicker thin films.

Second, our calculations show that the equilibrium lattice constant of the freestanding slabs is lower than the bulk one, as a consequence of surface stress relief. The effect is weak in the $\mathrm{BaO}$-terminated slabs, but significant in the $\mathrm{TiO}_{2}$ terminated ones. The surface stress is related to the fact that solid surfaces can minimize their energy by relaxing the positions of their atoms in the direction perpendicular to the surface, but are not able to do so in the lateral directions, since they are anchored by the underlying bulk lattice [34,35]. The surface layer of a solid is therefore usually stressed. Relief of this stress can lead the system to form surface reconstructions or adsorb molecules. We have shown here that the surface stress at perfect $\mathrm{BTO}(001)$ surfaces with $\mathrm{TiO}_{2}$ terminations is tensile, since it displaces the equilibrium lattice constant of the ultrathin film towards smaller values ( $3.91 \AA$ ) than the bulk one, i.e., towards compressive strain. In other words, the total stress tensor of the slab vanishes for negative (i.e., compressive) values of the bulk strain. The impact of the stress is probably overestimated because we study freestanding films, with surfaces on both sides. In real films, epitaxially grown on a substrate, the effect should be weaker.

\section{Electronic structure}

The electronic structure of the freestanding slabs we have calculated is characteristic of insulators: The Kohn-Sham band gap never vanishes, despite the use of the LDA, which is known to strongly underestimate the gap.

The Kohn-Sham band gaps $(\sim 2.0 \mathrm{eV})$ for the $\mathrm{BaO}$ terminated slabs are quite insensitive to the epitaxial strain and polarization direction. However, our calculations point out an important difference between the $c$ and $a a$ phases for the $\mathrm{TiO}_{2}$ termination: The Kohn-Sham band gap is found much smaller in the $c$ phase $(\approx 0.5 \mathrm{eV}$ for $a=3.80 \AA)$ than in the $a a$ phase $(\approx 1.4 \mathrm{eV}$ for $a=4.00 \AA)$. Two possible effects can be invoked to explain this difference of nearly $1 \mathrm{eV}$ :

(i) effect of a macroscopic potential (imperfectly compensated depolarizing field in the $c$ phase); and

(ii) effect of a microscopic potential (band bending which would depend on the polarization direction).

Figure 8 unambiguously shows that both $P^{+}$and $P^{-}$give rise to electronic surface states at the top of the valence band, located well above the bulk VBM in both cases. Figure 10 shows the projection of the density of states on each of the nine atomic layers of one domain of the slab in the $c$ phase: A clear effect of band bending can be observed at $P^{+}$ and $P^{-}$, mostly produced by a difference in the microscopic (Madelung) potential between the oxygen sites at the surface and in the bulk, $\Delta V_{\text {micro. }}$. The band-gap reduction from the $a a$ phase to the $c$ phase can be explained by the fact that $\left|\Delta V_{\text {micro }}\right|$ is higher in the $c$ phase than in the $a a$ phase, as a consequence of different ferroelectric distortions.

However, a very small energy difference of $\approx 0.2 \mathrm{eV}$ is observed between the VBM at $P^{+}$and $P^{-}$in the $c$ phase. In 


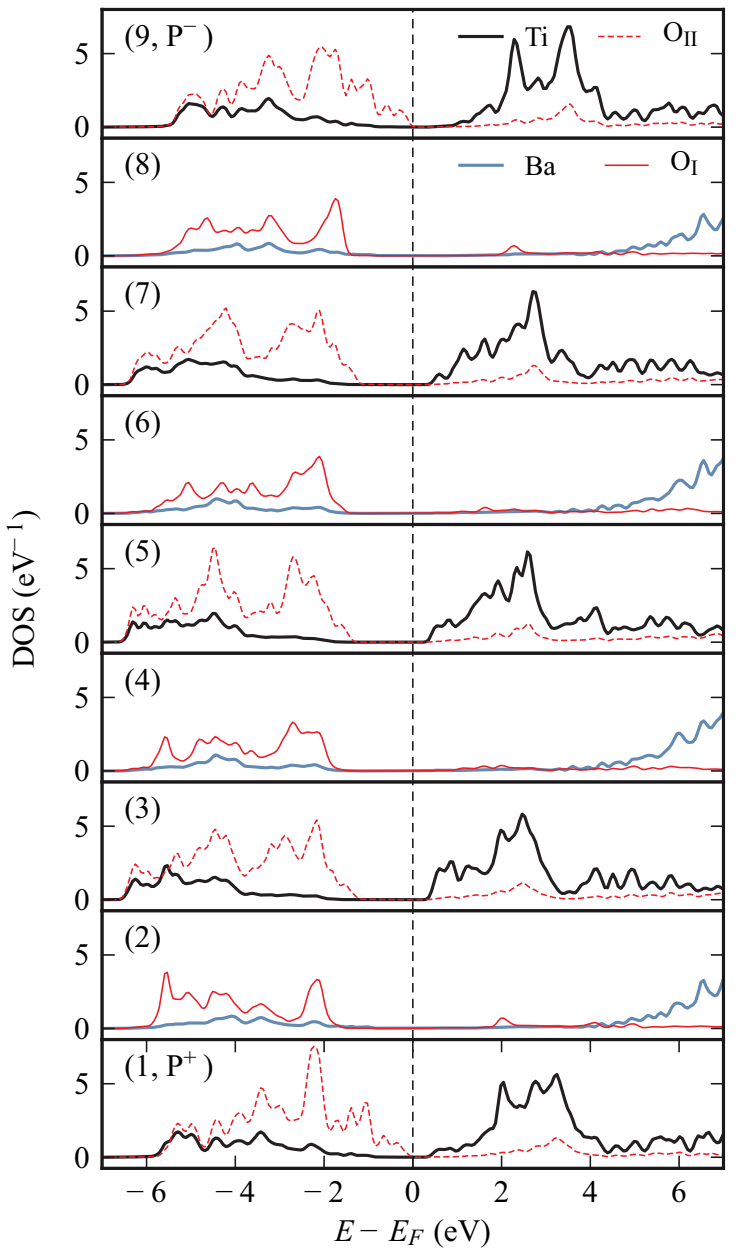

FIG. 10. (Color online) Electronic density of states of the $c$ phase projected on each of the nine atomic layers of one domain of the $\mathrm{TiO}_{2}$-terminated film, as computed with SIESTA. $N=6, a=3.80 \AA$. The atomic layers are indexed in the top left-hand corner of each plot.

order to determine whether this small difference is related or not to an imperfectly compensated depolarizing field, we plot, in Fig. 11, the profile of the macroscopic potential, computed as a double average, first over planes of fixed $z$, and then along $z$, by a convolution using a step function of length $l=4.0 \AA$ (the planar average has been performed over the whole supercell, and over each domain). A small potential difference $(\approx 0.3$ $\mathrm{eV})$ is present in one domain between $P^{+}$and $P^{-}$and is reversed in the other domain, suggesting the existence of a residual depolarizing field. Its effect on the electronic surface states (a few $0.1 \mathrm{eV}$ ) is, nevertheless, negligible with respect to that of the Madelung potential, which is responsible for a much larger shift $(\approx 1.5 \mathrm{eV})$.

In ionocovalent crystals, the existence of surface states at the VBM (and at the bottom of the conduction band) is usually related to a smaller Madelung potential at surface sites with respect to the bulk [36,37]. Our results suggest that this microscopic (Madelung) potential at the oxygen sites of the surface layer is strongly impacted by the additional, ferroelectric induced surface distortion. To confirm this point, we compute the density of states in the nonpolar $p$ phase for the $\mathrm{TiO}_{2}$ termination, and observe a very small band gap that is

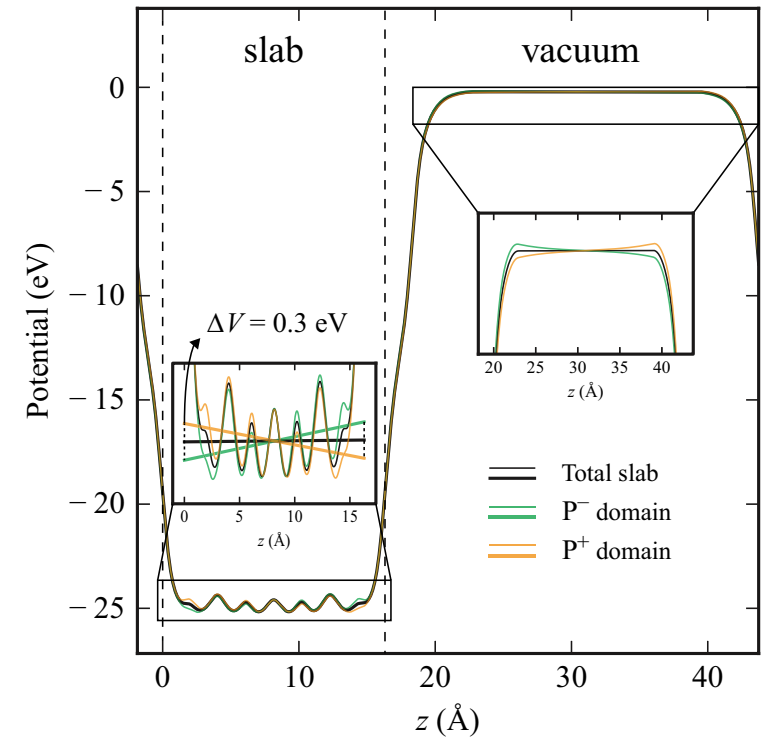

FIG. 11. (Color online) Macroscopic average of the total potential in the slab and in the vacuum as a function of $z(\AA)$, performed on the whole slab and on each domain (labeled according to the direction of the polarizarion at the top surface). $N=6, a=3.80 \AA$, vacuum thickness $\approx 30 \AA$ A. Left-hand-side inset: Zoom on the slab and linear fit of the macroscopic potential performed on the whole slab and on each domain. Right-hand-side inset: Zoom on the vacuum.

almost insensitive to the epitaxial strain $(0.4 \mathrm{eV}$ for $a=3.80 \AA$ and $0.7 \mathrm{eV}$ and $a=4.00 \AA$ ). In the $c$ phase, the ferroelectric displacements, superimposed to those of the natural rumpling, do not modify the band gap with respect to the $p$ phase, while in the $a a$ phase, they are responsible for an increase to $1.4 \mathrm{eV}$.

\section{CONCLUSION}

In this paper, we have investigated the structural, ferroelectric, and electronic properties of perfect $\mathrm{BaTiO}_{3}$ ultrathin films nine atomic layers thick $(\approx 1.6 \mathrm{~nm})$. The in-plane lattice constant has been varied to simulate the effect of epitaxial strain, and domain formation has been allowed for two, four, and six lattice constants wide supercells. We have found that configurations with polarization perpendicular to the surface are stabilized under compressive strain, while configurations with in-plane polarization (along [110]) are stabilized at larger lateral lattice constants. The transition between the two phases is shifted (with respect to bulk) towards compressive strain, as a consequence of the surface effects, and the equilibrium lattice constant of the $\mathrm{TiO}_{2}$-terminated slabs is lower than the bulk one, due to surface stress relief. We have determined that the most stable domain structure has a periodicity of two to four lattice constants, i.e., domains one to two lattice constants wide, depending on the surface termination.

The rumpling usually observed on oxide surfaces, with the anions moving outwards, is preserved, whatever the direction of the surface polarization. In the $c$ phase, we find that the polarization at the surface near the domain wall turns, forming closure domains in order to minimize the density of polarization charge, and thus the electrostatic energy. Finally, the system remains an electronic insulator 
in all the configurations studied. Significant reduction of the Kohn-Sham band gap with respect to the bulk is observed at the $\mathrm{TiO}_{2}$ termination with polarization perpendicular to the surface. This reduction occurs because the difference of Madelung potential between surface and bulk is stronger in the $c$ phase than in the $a a$ phase with $\mathrm{TiO}_{2}$ termination. Evidence for a small residual depolarizing field in the $c$ phase has been given, although its effect on the electronic surface states is small with respect to that of the Madelung potential.

Future work should include the effect of the slab size on the stability of the closure domains; in particular, although costly in terms of calculation time, we would like to check the $N=8$ pattern periodicity for the $\mathrm{BaO}$-terminated slab. In a further development, the next step of our study will be to include an oxygen vacancy in order to describe the interaction between internal chemical and physical screening mechanisms.

\section{ACKNOWLEDGMENTS}

This work was performed using the HPC resources from GENCI-CCRT/CINES (Grant No. 2013-097037). We gratefully acknowledge funding from the Agence National de la Recherche project ANR-12-IS04-0001-01 CHEM-SWITCH. $\mathrm{Ph}$. Ghosez is acknowledged for fruitful discussions.
[1] Y.-Y. Mi, G. Geneste, J. E. Rault, C. Matthieu, A. Pancotti, and N. Barrett, J. Phys.: Condens. Matter 24, 275901 (2012).

[2] R. V. Wang et al., Phys. Rev. Lett. 102, 047601 (2009).

[3] G. Geneste and B. Dkhil, Phys. Rev. B 79, 235420 (2009).

[4] M. Stengel, P. Aguado-Puente, N. A. Spaldin, and J. Junquera, Phys. Rev. B 83, 235112 (2011).

[5] J. Junquera and $\mathrm{Ph}$. Ghosez, Nature (London) 422, 506 (2003).

[6] P. Aguado-Puente and J. Junquera, Phys. Rev. Lett. 100, 177601 (2008).

[7] B. Meyer and D. Vanderbilt, Phys. Rev. B 63, 205426 (2001).

[8] M. Fechner, S. Ostanin, and I. Mertig, Phys. Rev. B 77, 094112 (2008).

[9] T. Shimada, S. Tomoda, and T. Kitamura, Phys. Rev. B 81, 144116 (2010).

[10] A. Schilling, T. B. Adams, R. M. Bowman, J. M. Gregg, G. Catalan, and J. F. Scott, Phys. Rev. B 74, 024115 (2006).

[11] G. Geneste, J. Phys.: Condens. Matter 23, 125901 (2011).

[12] W. Kohn and L. J. Sham, Phys. Rev. 140, A1133 (1965).

[13] X. Gonze, G.-M. Rignanese, M. Verstraete, J.-M. Beuken, Y. Pouillon, R. Caracas, F. Jollet, M. Torrent, G. Zerah, M. Mikami, Ph. Ghosez, M. Veithen, J.-Y. Raty, V. Olevano, F. Bruneval, L. Reining, R. Godby, G. Onida, D. R. Hamann, and D. C. Allan, Z. Kristallogr. 220, 558 (2005).

[14] J.-P. Perdew and Y. Wang, Phys. Rev. B 45, 13244 (1992).

[15] N. Troullier and J. L. Martins, Phys. Rev. B 43, 1993 (1991).

[16] Ph. Ghosez, Ph.D. thesis, Université Catholique de Louvain, 1997; J. Padilla, D. Vanderbilt, Phys. Rev. B 56, 1625 (1997).

[17] P. Ordejón, E. Artacho, and J. M. Soler, Phys. Rev. B 53, R10441(R) (1996).

[18] J. M. Soler, E. Artacho, J. D. Gale, A. García, J. Junquera, P. Ordejón, and D. Sánchez-Portal, J. Phys.: Condens. Matter 14, 2745 (2002).

[19] O. Dieguez, S. Tinte, A. Antons, C. Bungaro, J. B. Neaton, K. M. Rabe, and D. Vanderbilt, Phys. Rev. B 69, 212101 (2004).
[20] N. A. Pertsev, A. G. Zembilgotov, and A. K. Tagantsev, Phys. Rev. Lett. 80, 1988 (1998).

[21] B.-K. Lai, I. A. Kornev, L. Bellaiche, and G. J. Salamo, Appl. Phys. Lett. 86, 132904 (2005).

[22] O. Dieguez, K. M. Rabe, and D. Vanderbilt, Phys. Rev. B 72, 144101 (2005).

[23] B. Dupe, S. Prosandeev, G. Geneste, B. Dkhil, and L. Bellaiche, Phys. Rev. Lett. 106, 237601 (2011).

[24] G. Geneste, E. Bousquet, J. Junquera, and Ph. Ghosez, Appl. Phys. Lett. 88, 112906 (2006).

[25] P. Müller, A. Saùl, and F. Leroy, Adv. Nat. Sci.: Nanosci. Nanotechnol. 5, 013002 (2014).

[26] Ph. Ghosez, J.-P. Michenaud, and X. Gonze, Phys. Rev. B 58, 6224 (1998).

[27] J. Hlinka, T. Ostapchuk, E. Buixaderas, C. Kadlec, P. Kuzel, I. Gregora, J. Kroupa, M. Savinov, A. Klic, J. Drahokoupil, I. Etxebarria, and J. Dec, Phys. Rev. Lett. 112, 197601 (2014).

[28] A. K. Tagantsev, K. Vaideeswaran, S. B. Vakhrushev, A. V. Filimonov, R. G. Burkovsky, A. Shaganov, D. Andronikova, A. I. Rudskoy, A. Q. R. Baron, H. Uchiyama, D. Chernyshov, A. Bosak, Z. Ujma, K. Roleder, A. Majchrowski, J.-H. Ko, and N. Setter, Nat. Commun. 4, 2229 (2013).

[29] J. E. Rault, J. Dionot, C. Mathieu, V. Feyer, C. M. Schneider, G. Geneste, and N. Barrett, Phys. Rev. Lett. 111, 127602 (2013).

[30] G. Geneste, H. Dammak, M. Hayoun, and M. Thiercelin, Phys. Rev. B 87, 014113 (2013).

[31] W. Zhong and D. Vanderbilt, Phys. Rev. B 53, 5047 (1996).

[32] J. Iniguez and D. Vanderbilt, Phys. Rev. Lett. 89, 115503 (2002).

[33] B. Meyer and D. Vanderbilt, Phys. Rev. B 65, 104111 (2002).

[34] D. Vanderbilt, Phys. Rev. Lett. 59, 1456 (1987).

[35] R. J. Needs, Phys. Rev. Lett. 58, 53 (1987).

[36] C. Noguera, Physics and Chemistry at Oxide Surfaces (Cambridge University Press, Cambridge, UK, 1996).

[37] G. M. Vanacore, L. F. Zagonel, and N. Barrett, Surf. Sci. 604, 1674 (2010). 